\title{
Comparando os métodos paramétrico e não-paramétrico na determinação do valor crítico do teste estatístico de médias proposto por Hayter e Tsui
}

Suel Aparecida Mingoti

Fernando Augusto Alves Glória

Departamento de Estatística da UFMG

\begin{abstract}
Resumo
Neste artigo é feita uma comparação dos métodos paramétrico e não-paramétrico para determinação da constante $C_{f a}$, que é utilizada na proposta de Hayter e Tsui (1994) para controle de qualidade de processos multivariados. 0 método de Hayter e Tsui é uma alternativa ao $T^{2}$ de Hotelling, com a vantagem que identifica automaticamente quais variáveis são as responsáveis pela falta de controle do processo. Alguns processos multivariados de dimensões $\mathrm{p}=2$ e $\mathrm{p}=4$ foram simulados e os resultados mostraram que o método paramétrico é melhor que o não-paramétrico, mas para amostras de tamanhos maior ou igual a 5000 os dois métodos são equivalentes. Este resultado é contrário ao postulado por Hayter \& Tsui, que sugerem que o método nãoparamétrico pode ser adotado a partir de amostras de tamanho 500.
\end{abstract}

Palavras-chave

Processos multivariados, comparações múltiplas, controle de qualidade.

\section{Comparing the parametric and non-parametric methods for the determination of the critical value used in Hayter and Tsui's statistical test to compare means}

\begin{abstract}
In this paper a comparison between the parametric and the non-parametric methods used to find the constant $C_{B \alpha^{\prime}}$ which is part of the Hayter and Tsui (1994) methodology to control multivariate processes, is presented. The Hayter and Tsui method is an alternative to the Hotelling $T^{2}$ statistical test with the advantage that it identifies automatically which quality characteristic is responsible for the out-of-control situation. Some multivariate processes $p=2$ and $p=4$ dimensional were simulated and the results showed that the parametric method was better than the non-parametric one. However, for sample sizes higher ou equal to 5000 they were equivalent. The results go against the Hayter \& Tsui's who suggested that the non-parametric method could be adopted for sample sizes higher or equal to 500.
\end{abstract}

Key words

Multivariate processes, multiple comparisons, quality control. 


\section{INTRODUĈ̣̃O}

Em geral, os processos são monitorados através da observação de várias características de qualidade, variáveis estas que, na maioria das vezes, são correlacionadas entre si (MONTGOMERY, 2004). Embora o controle de qualidade do processo e a avaliação de sua capacidade possam ser feitos analisando-se cada característica separadamente através de gráficos de controle de Shewhart (COSTA et al., 2004) ou gráficos derivados, e do cálculo de índices de capacidade do tipo $C_{p}, C_{p k}, C_{p m}$, estas análises não levam em consideração a correlação natural existente entre as características de qualidade. Portanto, elas podem ser melhoradas se técnicas estatísticas multivariadas apropriadas forem utilizadas. atender-se à premissa de que o vetor aleatório que contém as características de qualidade que estão sendo avaliadas tenha distribuição normal multivariada (JOHNSON \& WICHERN, 2002), embora o teste seja relativamente robusto à falta de normalidade para distribuições aproximadamente simétricas (MARDIA, 1974).

O uso de gráficos de controle multivariados é recente e algumas referências interessantes de aplicação são: Mason \& Young (2002), Mason, Chou et al. (2001), Mason, Tracy et al. (1997), Nomikos \& MacGregor (1995), dentre outros. Uma possível crítica ao uso do teste $T^{2}$ Hotelling para avaliar o processo vem do fato de que no momento em que a hipótese nula é rejeitada, torna-se necessário identificar as características de qualidade responsáveis pela sua rejeição, o que muitas vezes é feito através de gráficos de Shewhart para cada variável isoladamente, corrigindo-se ou não os níveis de significância dos testes estatísticos feitos separada-

No que tange ao controle de processos via construção de gráficos, a extensão para o caso multivariado pode ser feita considerando-se testes estatísticos apropriados para testar vetores de médias, já que o gráfico de controle pode ser visto como um teste estatístico de hipóteses no qual a hipótese nula é a de que o processo está "sob controle", isto é, as características de qualidade avaliadas estão com suas médias e estruturas de variabilidades controladas de acordo com os parâmetros especificados para o processo, enquanto que a hipótese alternativa é a de que o processo está fora de controle em relação a alguns desses parâmetros.

Dentro da área de testes estatísticos de hipóteses multivariados encontra-se o teste $T^{2}$ de Hotelling (1947) para comparação de vetores de médias populacionais. Assim, são encontrados vários artigos na literatura propondo o uso deste teste como uma forma de avaliar o processo. A partir do teste $T^{2}$ de Hotelling, constrói-se o elipsóide de confiança que permite verificar se o processo está ou não sob controle considerando-se todas as características simultaneamente. Também é possível construir elipsóides de predição para valores futuros do processo (JOHNSON \& WICHERN, 2002). Um outro gráfico que pode ser feito a partir do teste de Hotelling é o do qui-quadrado, que é uma forma de transformar a informação multivariada numa estatística unidimensional cujos valores podem ser grafados, estabelecendo-se, então, um limite superior de controle (MASON \& YOUNG, 2002). Para que o teste de Hotelling tenha validade é necessário mente para a média de cada característica. Correções dos níveis de significância como as usadas em testes de comparações múltiplas podem ser utilizadas. A mais comum é a de Bonferroni (JOHNSON \& WICHERN, 2002; HAYTER \& TSUI, 1994), na qual o nível de significância para cada variável é escolhido como igual a $\frac{\alpha}{p}$, onde $p$ é o número de características de qualidade avaliadas e a estatística $t$-Student é utilizada como referência para rejeição ou não da hipótese nula de controle para cada variável isoladamente. Alt (1985) e Doganaksoy et al. (1991) sugerem que ao invés da estatística $t$-Student o valor de referência delimitador da região de rejeição da hipótese nula seja proveniente da estatística qui-quadrado. Estas correções, no entanto, não levam em consideração a correlação entre as características de qualidade, enfraquecendo o poder e a sensibilidade do teste. É possível, por exemplo, que o teste $T^{2}$ Hotelling indique que o processo está fora de controle e que não se detecte através destas comparações múltiplas quais das variáveis são responsáveis pelo fato. Para evitar este problema, Doganaksoy et al. (1991) sugerem que se trabalhe com um nível de significância global $\alpha$ maior do que o usual. Outra alternativa é trabalhar-se com a desigualdade de Dunn-Sidak (DUNN, 1958; SIDAK, 1967) ao invés da desigualdade de Bonferrroni para a delimitação do nível de significância dos testes de comparações múltiplas; isto é, no lugar de $\frac{\alpha}{p}$ seria utilizado o valor $1-(1-\alpha)^{\frac{1}{p}}$, o que 
teoricamente leva a um procedimento mais poderoso que o de Bonferroni em vista do fato de que $1-(1-\alpha)^{\frac{1}{p}}>\frac{\alpha}{p}$ para qualquer $p$ e $\alpha$, sendo $\alpha$ o nível de significância global dos testes. Na prática, no entanto, as correções de Bonferroni e de Dunn-Sidak levam a resultados semelhantes (HAYTER \& TSUI, 1994).

Uma alternativa bastante interessante, que propõe uma correção nas comparações múltiplas quando a hipótese nula de controle estatístico do processo é rejeitada, surgiu no artigo de Hayter \& Tsui (1994). Estes autores propõem que intervalos de confiança (ou gráficos de controle) sejam feitos separadamente para cada variável mas de modo que a abertura dos limites de confiança leve em consideração a correlação existente entre as variáveis medidas. O processo de construção dos intervalos de confiança assegura que o nível de confiança de cada intervalo seja mantido constante e igual ao nível de confiança global fixado inicialmente para o teste de comparações múltiplas. Além disso, permite que se tenha uma regra que automaticamente identifica as variáveis causadoras da rejeição da hipótese nula de controle estatístico multivariado, ou seja, a identificação das variáveis responsáveis pela situação de falta de controle do processo. Esta identificação rápida das variáveis causadoras dos problemas no processo seria o grande motivador para que o usuário passasse a utilizar a proposta de Hayter \& Tsui (1994) ao invés do teste de $T^{2}$ Hotelling combinado com os intervalos de confiança de Bonferroni ou de DunnSidak, uma vez que este novo processo gera intervalos de menor amplitude, como mostrado pelos autores do artigo.

Em Hayter e Tsui (1994) são propostos dois métodos de se obter a constante $C_{R \alpha}$ que está relacionada com a construção dos intervalos de confiança usados para monitorar o processo. O primeiro é um método paramétrico que envolve a simulação de observações de uma distribuição normal multivariada. De acordo com Hayter e Tsui, a partir de uma amostra do processo estima-se o vetor de médias e a matriz de correlação do processo. Uma amostra de $\mathrm{N}$ observações seria então gerada de uma distribuição normal p-variada com os parâmetros iguais às estimativas obtidas. A partir desta amostra simulada, o valor da constante $C_{R \alpha}$ seria obtido. Os autores indicam que $k=100000$ observações simuladas seria o número necessário para a implementação deste método. O segundo é um método não-paramétrico que se fundamenta apenas no uso dos dados amostrais originais observados para a determinação do valor de $C_{R \alpha}$, sem envolver qualquer processo de simulação.
Hayter e Tsui sugerem que, se a amostra original tiver cerca de 500 observações, o método não-paramétrico poderá ser adotado para a determinação da constante $C_{R \alpha}$. A constante $C_{R \alpha}$ também tem um papel importante no cálculo dos coeficientes de capacidade multivariados propostos por Mingoti \& Glória (2003), que são modificações do índice de capacidade proposto por Chen (1994).

O objetivo deste artigo é apresentar uma comparação entre os dois métodos propostos por Hayter e Tsui para a determinação da constante $C_{R \alpha}$ com o intuito de definir a partir de qual tamanho de amostra os dois métodos produziriam resultados semelhantes. A distribuição considerada como base para esse estudo é a normal multivariada.

\section{HAYTER \& TSUI (1994): CORREC̣ÃO DOS LIMITES DE CONTROLE}

Seja $X=\left(X_{1} X_{2} \ldots X_{p}\right)^{\prime}$ o vetor contendo as características de qualidade de interesse, $X$ tendo distribuição normal p-variada com vetor de médias $\mu_{0}=\left(\mu_{0}{ }^{1} \mu_{0}{ }^{2} \ldots \mu_{0}{ }^{p}\right)^{\prime} \mathrm{e}$ matriz de covariâncias $\Sigma_{p x x}$. De acordo com Hayter e Tsui (1994), para cada variável $X_{i}$ os limites de confiança de (1- $\alpha) 100 \%, 0<\alpha<1$, são dados pela equação:

$$
\operatorname{Prob}\left[\left[\left|\frac{X_{i}-\mu_{i}^{0}}{\sigma_{i}}\right| \leq C_{R \alpha}, \forall i=1,2, \ldots, p\right]=1-\alpha\right.
$$

o que significa dizer que a probabilidade de que o intervalo: $\left[X_{i} \pm \sigma_{i} C_{R \alpha}\right]$ contenha o valor verdadeiro $\mu_{i}^{0}$ para todo $i, i=1,2, \ldots, p$, é igual a (1- $\alpha)$. A escolha do valor crítico depende da matriz de correlação teórica $P_{p x p}$ do vetor aleatório $X$. Desse modo, o processo é considerado como fora de controle quando:

$M=\max \left\{\left|\frac{X_{i}-\mu_{i}^{0}}{\sigma_{i}}\right|, i=1,2, \ldots, p\right\}>C_{R \alpha}$

O valor de $C_{R \alpha}$ é obtido através de um algoritmo que envolve a simulação de amostras de uma população normal p-variada com vetor de médias zero e matriz de covariâncias $P_{p x p}$, que na prática é estimada pela matriz de correlação amostral das variáveis observadas e é denotada por $R_{p x p}$ (JOHNSON \& WICHERN, 2002). Os passos deste algoritmo são mostrados na Figura 1. É importante observar que na proposta de Hayter e Tsui a estrutura de correlação do vetor aleatório $X$ afeta todos os intervalos simultaneamente, ao contrário dos intervalos simultâneos de Bonferroni, nos quais apenas o valor de 
referência da distribuição t-Student, utilizada para a construção dos intervalos, é alterado de modo a manterse o nível de significância global de comparação requerido a priori para o teste.

Hayter e Tsui (1994) sugerem que sejam geradas $k=100000$ observações de uma distribuição normal p-variada para a determinação de $C_{R \alpha}$ com grande precisão e mostram que os intervalos de confiança assim construídos são melhores que os intervalos de Bonferroni.

Dados da literatura indicam que a constante $C_{R \alpha}$ pode ser obtida, para casos normais bi-variados $(p=2)$, através de valores tabelados, segundo o artigo de Bechhofer e Dunnet (1988). A obtenção dessa constante, no caso de normalidade, para mais que duas variáveis $(p>2)$, pode ser feita através de integração numérica, algo complicado, uma vez que a determinação da constante $C_{R \alpha}$ envolve a determinação da distribuição do máximo do vetor aleatório $X$. Daí a importância dos métodos de obtenção de $C_{R \alpha}$ propostos por Hayter e Tsui (1994).

Quando o tamanho da amostra é grande, o algoritmo da Figura 1 pode ser modificado, sendo a função distribuição empírica da estatística $M$ calculada usando-se apenas os $n$ vetores observados da amostra original e não mais através de uma simulação da distribuição normal p-variada, como mostra a Figura 2. Neste caso, o método independe do fato de o vetor $X$ ter ou não uma distribuição normal, sendo, portanto, um método nãoparamétrico. No entanto, segundo Hayter e Tsui seria necessário ter-se uma amostra de no mínimo 500 observações para poder aplicar este método, algo nem sempre disponível em muitos problemas práticos. Da mesma forma que no método paramétrico, dada uma nova observação $X=\left(X_{1}, X_{2}, \ldots, X_{p}\right)$ ' do processo calcula-se a estatística $M$ e o processo, é declarado como fora de controle se $M>C_{R \alpha}$. As variáveis $X_{j}$ tais que $\left(\left|X_{j}-\bar{X}_{j}\right| / \sqrt{S_{j j}}\right)>C_{R \alpha}$ são as responsáveis pela falta de controle do processo, onde $\bar{X}_{j}$ e $\mathrm{S}_{j j}$ denotam respectivamente a média e a variância amostral da característica de qualidade $X_{j}$.

\section{COMPARAC̣ÃO DOS MÉTODOS VIA SIMULAC̣̃̃O}

Nesta seção apresentam-se os resultados da comparação dos métodos paramétrico e não-paramétrico para obtenção da constante $C_{R \alpha}$. Em Mingoti e Glória (2003) foi mostrado que para o caso paramétrico, amostras simuladas de tamanhos iguais a $k=10000$ já forneciam boas estimativas para a constante $C_{R \alpha}$. Sendo assim, para efeito da comparação que será apresentada neste artigo, o valor $k=10000$ será fixado para o cálculo da constante $C_{R \alpha}$ no método paramétrico e os valores considerados para o tamanho $\mathrm{n}$ das amostras que serão usadas para aplicação do método não-paramétrico serão: 50, 100, 500, 1000, 5000, 10000 e 100000 , com o objetivo de avaliar a partir de qual tamanho de amostra os dois métodos se aproximam em relação às estimativas obtidas para o valor da constante $C_{R \alpha^{\circ}}$. $O$ processo de simulação foi desenvolvido de acordo com os passos descritos na Figura 3. A programação computacional foi implementada no software estatístico S-Plus.

Figura 1: Algoritmo usado para encontrar a constante $C_{R \alpha}-$ Método paramétrico.

1. Gerar um grande número $k$ de vetores de observações de uma normal $p$-variada padronizada, isto é, com vetor de médias zero e matriz de covariâncias $P_{p x p}$, denotados por: $Z^{1}, Z^{2}, \ldots, Z^{k}$.

2. Calcular a estatística $M$ para cada um dos vetores $Z^{i}=\left(Z_{1}^{i}, Z_{2}^{i}, \ldots, Z_{p}^{i}\right)$ gerados no passo 1 , isto é, para todo $i=1,2, \ldots, k$, calcular:

$$
M^{i}=\max \left\{\left|Z_{j}^{i}\right|, j=1,2, \ldots, p\right\}
$$

3. Encontrar a ordenada do percentil de ordem $(1-\alpha)$ da amostra $\left(M^{1}, M^{2}, \ldots, M^{k}\right) \mathrm{e}$ usar o valor encontrado como sendo o valor crítico $C_{R \alpha}$. 
Para efeito de comparação, os processos simulados foram os mesmos tratados no artigo de Hayter e Tsui (1994).

\section{Simulação para o caso bivariado $(p=2)$}

O primeiro processo simulado foi o primeiro exemplo apresentado no artigo de Hayter e Tsui (1994) e que tem os seguintes parâmetros:

vetor de médias populacional: $\mu^{0}=\left[\begin{array}{ll}265 & 470\end{array}\right]$;

matriz de covariâncias populacional: $\Sigma^{0}=\left[\begin{array}{cc}10 & 6,6 \\ 6,6 & 12,1\end{array}\right]$; matriz de correlação populacional: $\rho^{0}=\left[\begin{array}{cc}1 & 0,6 \\ 0,6 & 1\end{array}\right]$.

Das tabelas de Bechhofer e Dunnet (1988), sabe-se que o valor da constante $C_{R \alpha}$ para p=2 e $\alpha=0,05$ é 2,199. Dessa forma, fizemos as comparações em relação a este valor.

Análise dos resultados: caso bivariado

A Tabela 1 apresenta os resultados gerais obtidos das N=100 repetições do algoritmo descrito na Figura 3, para os dois métodos de determinação da constante $C_{R \alpha}$, chamados aqui de método I (paramético) e método II (não-paramétrico). Pode-se observar que para $\mathrm{n}=50 \mathrm{o}$ método I foi o que mais se aproximou do verdadeiro valor da constante $C_{R \alpha}$, fornecendo também a menor variabilidade entre os 100 valores de $C_{R \alpha}$ obtidos na simulação ( a dispersão do método I é aproximadamente 8,5 vezes menor que a do método II). O método II fornece valores com maiores erros em relação ao valor tabelado da constante $C_{R \alpha}$ para p=2. A Figura 4 apresenta os gráficos da função distribuição empírica e os boxplots (JOHNSON \& BATTACHARRYYA, 2001) dos valores de $C_{R \alpha}$ obtidos pelos dois métodos para $\mathrm{n}=50$. Pode-se observar uma diferença acentuada entre os valores obtidos pelos dois métodos, uma vez que as curvas da distribuição empírica apresentam comportamentos bem diferentes. Isto é causado principalmente pela diferença na variabilidade dos resultados como evidenciado no box-plot, que mostra que a dispersão do método II é bem maior que a do método I. Pela Tabela 1 e Figura 5, observa-se o mesmo comportamento de resultados para amostras de tamanho igual a 100 .

Figura 2: Algoritmo usado para encontrar a constante $C_{R \alpha}-$ Método não-paramétrico.

Sejam $X^{1}, X^{2}, \ldots, X^{n}$ os vetores que representam uma amostra aleatória de tamanho $n$ do vetor $X$ de $p$ características de qualidade do processo, isto é, $X^{i}=\left(X_{1}^{i} X_{2}^{i} \ldots X_{p}^{i}\right)^{\prime}$.

1. Calcula-se o vetor de médias amostral $\bar{X}$ e a matriz de covariância amostral $S$;

2. Calcula-se a estatística $M$ para cada um dos vetores $X^{i}$ do passo 1 , isto é, para todo $\mathrm{i}=1,2, \ldots, n$, calcula-se:

$$
M^{i}=\max _{1 \leq j \leq p}\left\{\frac{\left|X_{j}^{i}-\bar{X}_{j}\right|}{\sqrt{S_{j j}}}\right\}
$$

onde $\bar{X}_{j}$ e $S_{j j}$ denotam respectivamente a média e a variância amostral da característica de qualidade $X_{j}$.

3. Encontrar a ordenada do percentil de ordem $(1-\alpha)$ da amostra $\left(M^{1}, M^{2}, \ldots, M^{n}\right)$ e usa-se o valor encontrado como sendo o valor crítico $C_{R \alpha}$. 
A Figura 6 apresenta os gráficos da função distribuição empírica e os box-plots dos valores de $C_{R \alpha}$ para $\mathrm{n}=500$. Observa-se que o desempenho do método I melhora em relação aos casos de $n=50$ e100 e que este continua a ter um melhor desempenho que o método II. No entanto, há uma substancial melhora nos valores fornecidos pelo método II, que teve sua média bastante próxima ao valor de 2,199 com uma dispersão menor do que os correspondentes valores obtidos para $n=50$ e 100 . Apesar disso, o método II produziu resultados ainda bastante diferentes dos obtidos pelo método I, como mostra a Figura 6. Alguns valores atípicos aparecem no método I, mas este continua a ser preferido em relação ao método II.

Quando o tamanho da amostra aumenta para $n=1000$ o método I continuou fornecendo a menor dispersão (ver Tabela 1 e Figura 7) e sua distribuição ainda continua sendo diferente da distribuição do método II. No entanto, as dispersões dos dois métodos são mais seme- lhantes do que nos casos de $\mathrm{n}=50,100$ e 500 .

Para amostras de tamanhos $n=5000,10000$ e 100000 os resultados dos dois métodos em termos médios foram muito parecidos (ver Tabela 1). Os valores fornecidos para a constante são praticamente iguais ao valor real tabelado fornecido por Bechhofer e Dunnet (1988). Para esses três tamanhos de amostra o método II produziu menor dispersão que o método I. Deste modo, para amostras de tamanho $\mathrm{n}$ maiores ou iguais a 5000 pode-se concluir que o método II é tão bom quanto o método I para obtenção da constante e tem menor dispersão no caso de normalidade. As funções distribuições empíricas apresentaram comportamentos muito semelhantes para $\mathrm{N}=5000$ e 10000 (ver Figuras 8 e 9), assim como as dispersões observadas dos dois métodos. Devido à dispersão do método II quando $\mathrm{N}=100000$ ser bem menor que a do método I (aproximadamente 6 vezes), as distribuições empíricas, nesse caso, não foram semelhantes (ver Figura 10).

Figura 3: Algoritmo usado para a simulação de dados e avaliação dos métodos de estimação de $\boldsymbol{C}_{\boldsymbol{R} \alpha}$.

Passo 1. Gera-se uma amostra aleatória de tamanho $n$ de uma distribuição normal p-variada com vetor de médias $\mu^{0}$ e matriz de covariância $\Sigma^{0}$. Calcula-se o vetor de médias amostrais $\bar{X}$, a matriz de covariância amostral $S$ e a matriz de correlação amostral $R$.

Passo 2. Utilizando-se a matriz de correlação amostral $R$ obtida no passo 1 , aplica-se o método paramétrico para encontrar a constante $C_{R \alpha}$ de acordo com o algoritmo da Figura 1.

Passo 3. Utilizando-se a amostra gerada no passo 1, aplica-se o método não-paramétrico para encontrar a constante $C_{R \alpha}$ de acordo com o algoritmo da Figura 2.

Passo 4. Repetem-se os passos de (1) a (3) N=100 vezes. Assim, obtêm-se 100 valores da constante $C_{R \alpha}$ provenientes dos dois métodos investigados.

Passo 5. Para cada um dos dois métodos calculam-se as estatísticas descritivas básicas e gráficos como o box-plot e o da função de densidade de probabilidade empírica acumulada, que mostram o comportamento dos valores obtidos da constante $C_{R \alpha}$.

Nota: Para cada amostra, a matriz de correlação amostral foi comparada com a matriz de correlação teórica para verificar se o processo de geração estava produzindo dados de acordo com a estrutura de correlação teórica postulada. 
Tabela 1: Comparação dos métodos de obtenção da constante $C_{R \alpha}$ para vários tamanhos de amostras.

\begin{tabular}{|c|c|c|c|c|c|}
\hline & & $\mathbf{n}=\mathbf{5 0}$ & & & \\
\hline Método & Média & Desvio-padrão & Mínimo & Mediana & Máximo \\
\hline Método I & 2,1987 & 0,0204 & 2,1471 & 2,2013 & 2,2415 \\
\hline \multirow[t]{2}{*}{ Método II } & 2,1505 & 0,1722 & 1,7956 & 2,1418 & 2,6561 \\
\hline & & $n=100$ & & & \\
\hline Método & Média & Desvio-padrão & Mínimo & Mediana & Máximo \\
\hline Método I & 2,1988 & 0,0202 & 2,1546 & 2,2002 & 2,2623 \\
\hline \multirow[t]{2}{*}{ Método II } & 2,1259 & 0,1163 & 1,8768 & 2,1277 & 2,4583 \\
\hline & & $\mathrm{n}=\mathbf{5 0 0}$ & & & \\
\hline Método & Média & Desvio-padrão & Mínimo & Mediana & Máximo \\
\hline Método I & 2,2021 & 0,0177 & 2,1552 & 2,2032 & 2,2362 \\
\hline \multirow[t]{2}{*}{ Método II } & 2,1847 & 0,0582 & 2,0175 & 2,1789 & 2,3460 \\
\hline & & $n=1000$ & & & \\
\hline Método & Média & Desvio-padrão & Mínimo & Mediana & Máximo \\
\hline Método I & 2,1987 & 0,0189 & 2,1396 & 2,1986 & 2,2400 \\
\hline \multirow[t]{2}{*}{ Método II } & 2,1941 & 0,0437 & 2,1052 & 2,1912 & 2,3115 \\
\hline & & $\mathbf{n}=\mathbf{5 0 0 0}$ & & & \\
\hline Método & Média & Desvio-padrão & Mínimo & Mediana & Máximo \\
\hline Método I & 2,1995 & 0,0206 & 2,1594 & 2,1995 & 2,2551 \\
\hline \multirow[t]{2}{*}{ Método II } & 2,1971 & 0,0172 & 2,1567 & 2,1966 & 2,2336 \\
\hline & & $n=10000$ & & & \\
\hline Método & Média & Desvio-padrão & Mínimo & Mediana & Máximo \\
\hline Método I & 2,2002 & 0,0182 & 2,1614 & 2,2000 & 2,2442 \\
\hline \multirow[t]{2}{*}{ Método II } & 2,1962 & 0,0133 & 2,1679 & 2,1953 & 2,2297 \\
\hline & & $n=100000$ & & & \\
\hline Método & Média & Desvio-padrão & Mínimo & Mediana & Máximo \\
\hline Método I & 2,1980 & 0,0188 & 2,1348 & 2,2000 & 2,2407 \\
\hline Método II & 2,1991 & 0,00355 & 2,1871 & 2,1993 & 2,2083 \\
\hline
\end{tabular}

Figura 4: Comparação entre os métodos I e II para amostras de tamanho $50(\alpha=0,05)$.

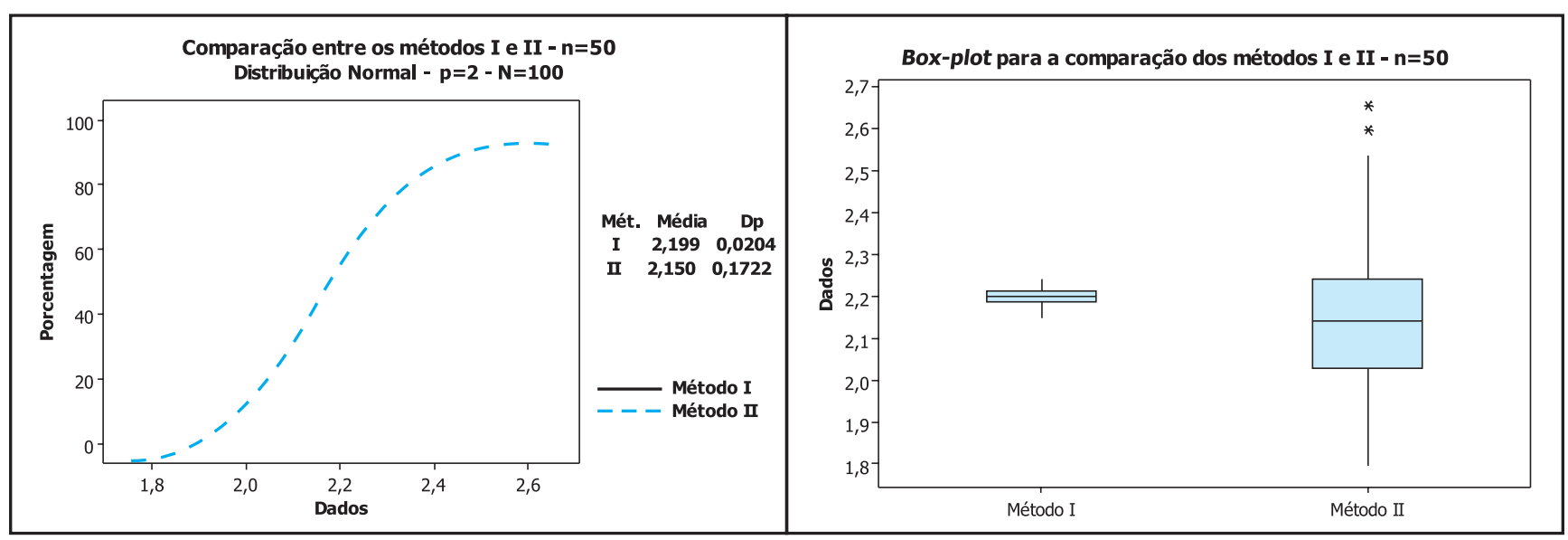


Figura 5: Comparação entre os métodos I e II para amostras de tamanho $100(\alpha=0,05)$.

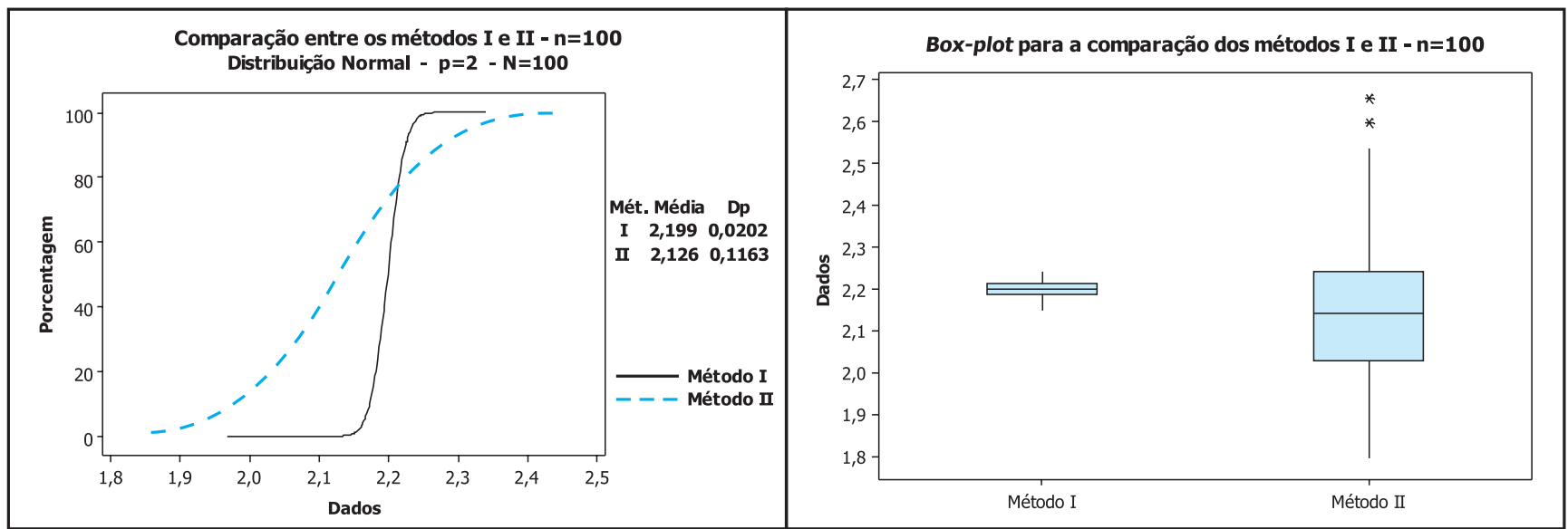

Figura 6: Comparação entre os métodos I e II para amostras de tamanho $500(\alpha=0,05)$.

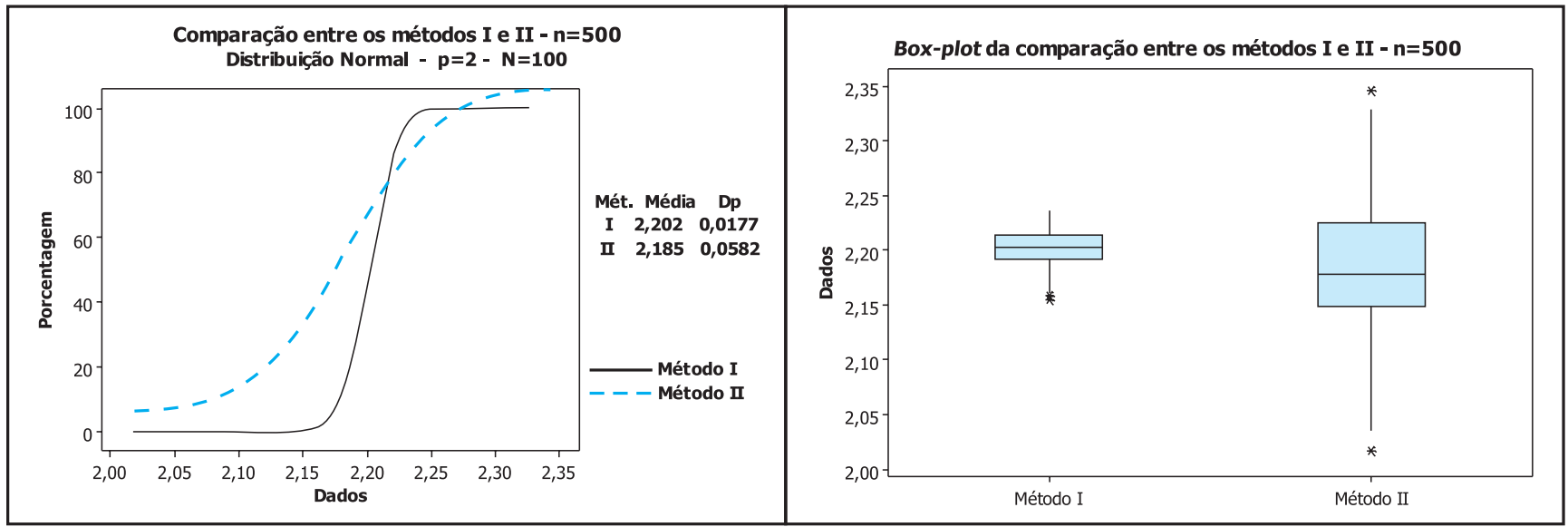

Figura 7: Comparação entre os Métodos I e II para amostras de tamanho $1000(\alpha=0,05)$.

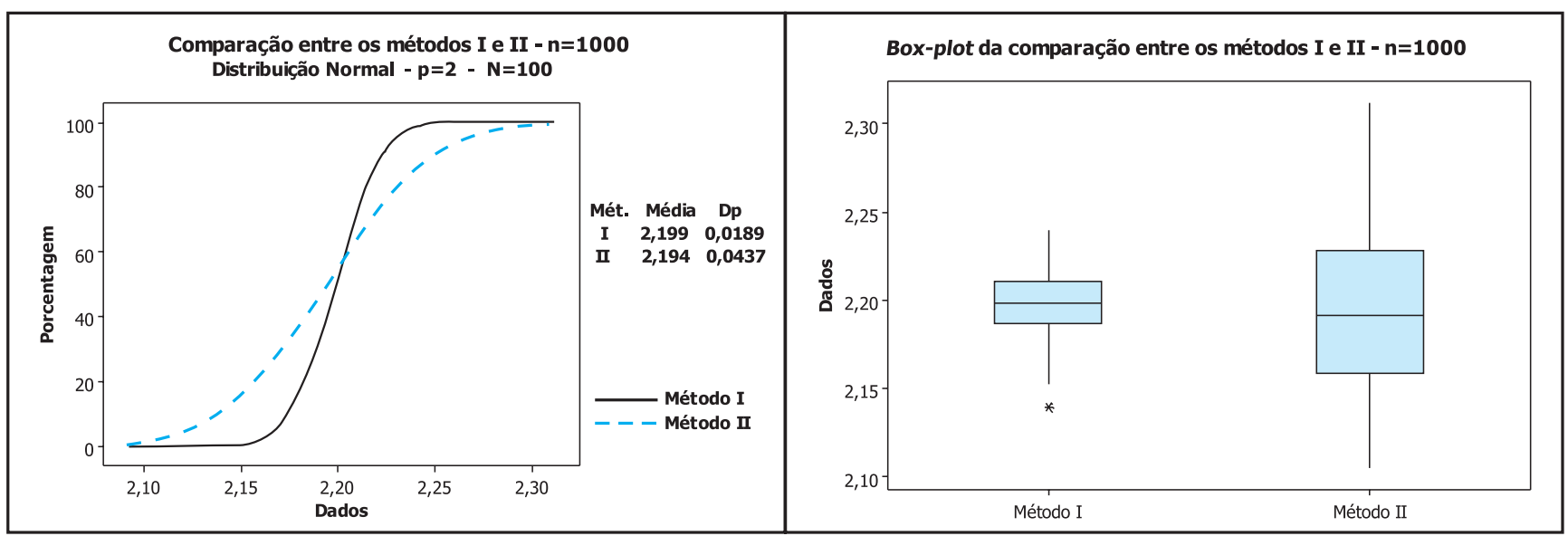


Figura 8: Comparação entre os métodos I e II para amostras de tamanho $5000(\alpha=0,05)$.
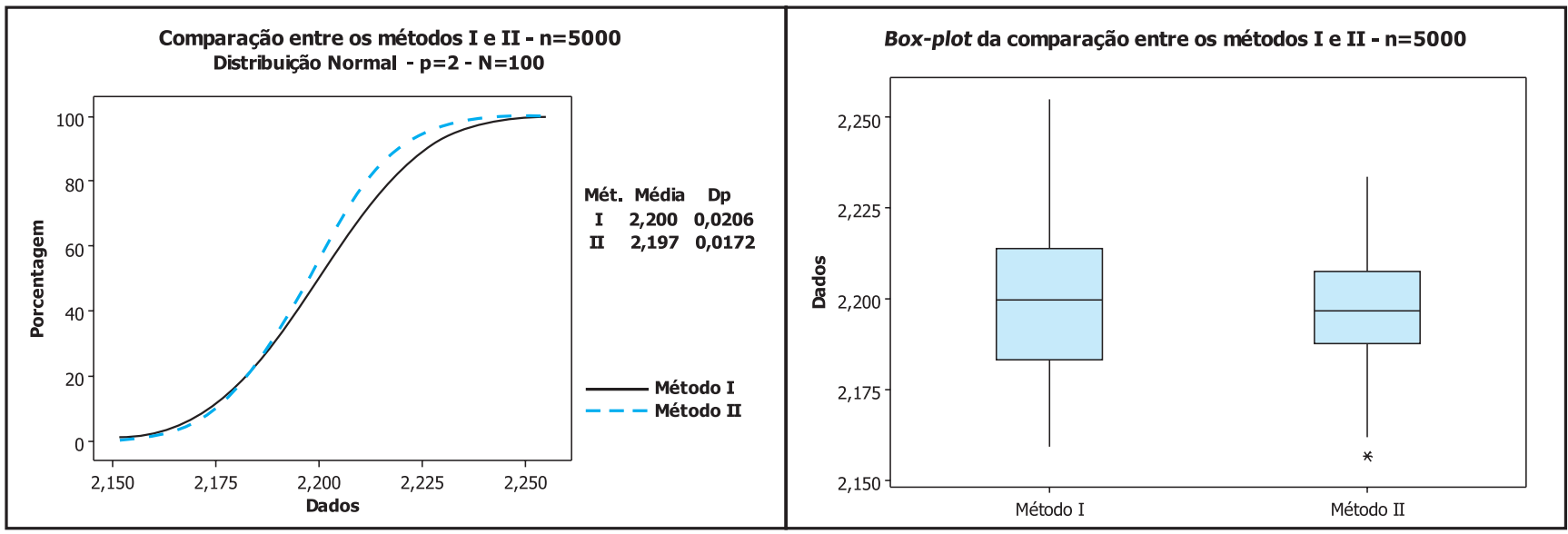

Figura 9: Comparação entre os Métodos I e II para amostras de tamanho $10000(\alpha=0,05)$.

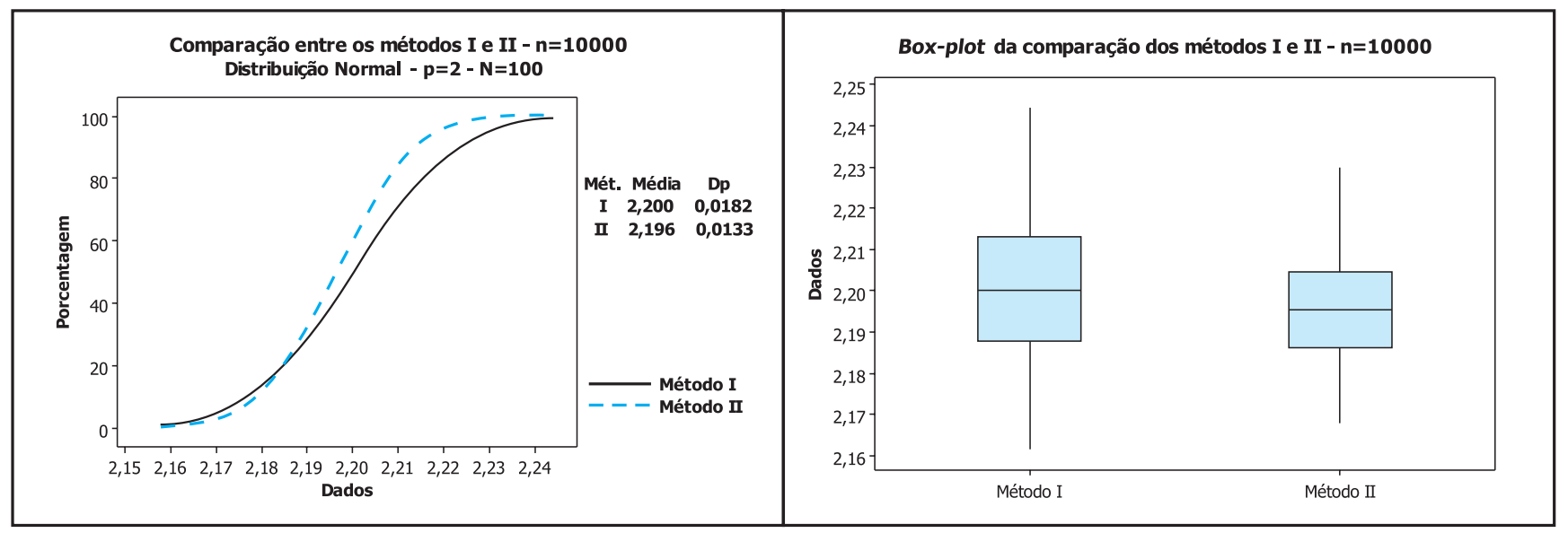

Figura 10: Comparação dos métodos I e II para amostras de tamanho $100000(\alpha=0,05)$.

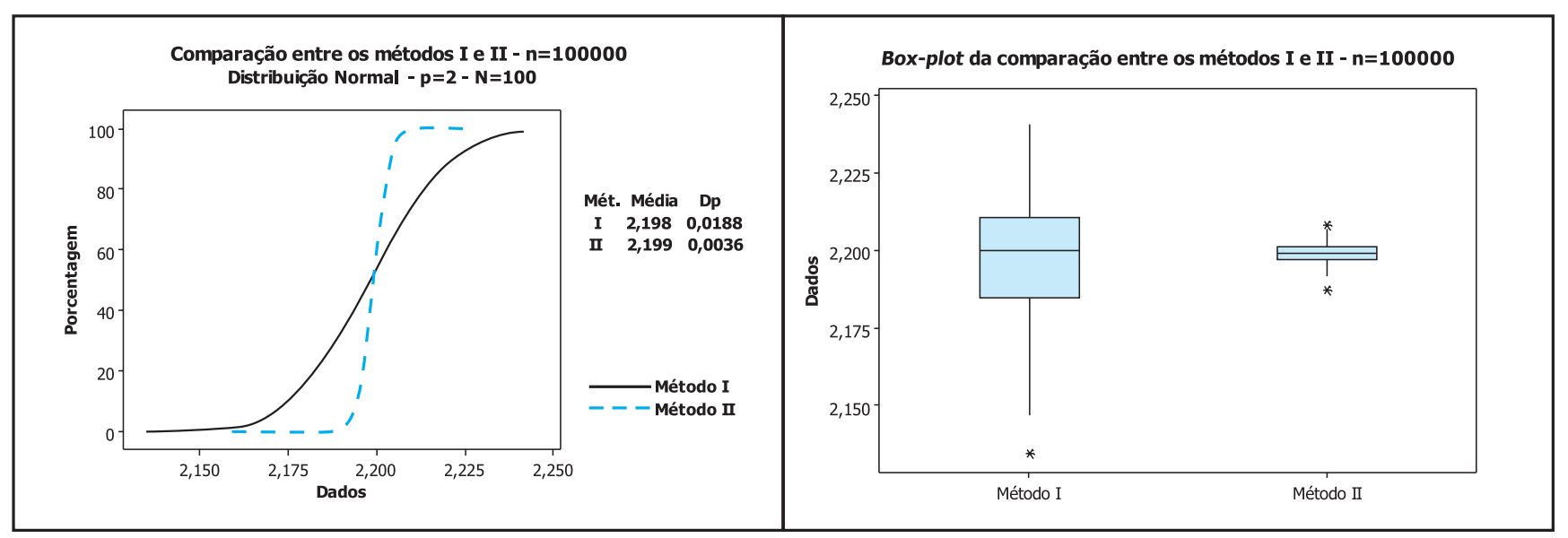


Para todos os tamanhos de amostra considerados os valores de média e mediana de $C_{R \alpha}$ foram similares, o que indica que a distribuição de valores de $C_{R \alpha}$ é aproximadamente simétrica.

Com a finalidade de se avaliar o processo de geração dos dados normais multivariados usados nas simulações, para cada uma das 100 amostras aleatórias geradas, a matriz de correlação amostral foi comparada com a matriz de correlação populacional. A Tabela 2 mostra algumas medidas descritivas das estimativas do coeficiente de correlação para cada tamanho de amostra. Os resultados mostram que as amostras geradas produziram valores de correlação bastante próximos da correlação populacional, que é igual a 0,60 .

\section{Simulação para o caso $p=4$}

Nesta seção mostramos os resultados da simulação de um outro exemplo contido no artigo de Hayter e Tsui (1994) para $\mathrm{p}=4$. Os parâmetros do processo são:

vetor de médias: $\mu^{0}=\left[\begin{array}{llll}0 & 0 & 0 & 0\end{array}\right]$;

matriz de covariância populacional:

$\Sigma^{0}=\left[\begin{array}{cccc}102,74 & 88,67 & 67,04 & 54,06 \\ 88,67 & 142,74 & 86,56 & 80,03 \\ 67,04 & 86,56 & 84,57 & 69,42 \\ 54,06 & 80,03 & 69,42 & 99,06\end{array}\right] ;$

matriz de correlação populacional:

$\rho^{0}=\left[\begin{array}{cccc}1,00000 & 0,732207 & 0,719211 & 0,535867 \\ 0,732207 & 1,00000 & 0,787837 & 0,673024 \\ 0,719211 & 0,787837 & 1,000000 & 0,758451 \\ 0,535867 & 0,673024 & 0,758451 & 1,000000\end{array}\right]$.

No artigo de Hayter e Tsui (1994) o valor da constante $C_{R \alpha}$ para este processo com $\alpha=0,05$ é igual a 2,37. Dessa forma, fizemos as comparações em relação a este valor. Ao contrário do exemplo tratado anteriormente, mostramos apenas os gráficos da função de distribuição empírica (Figuras 11 e 12). Como se pode observar, a partir de amostras de tamanho 5000, o método II forneceu melhores valores para a estimativa da constante do que o método I. Pode-se verificar que para $n=5000$ as curvas dos dois métodos são praticamente iguais e fornecem um valor médio da constante igual a 2,371 com desvios padrões similares. É interessante observar que, no geral, para todos os tamanhos de amostra considerados o comportamento da dispersão da distribuição de para $\mathrm{p}=2$ foi semelhante ao observado para $\mathrm{p}=4$. Para valores de $n$ pequenos $(\mathrm{n}=50,100)$, o método I foi bem mais preciso que o método II. Para valores de $\mathrm{n}=500,1000$ e 5000, as discrepâncias entre as dispersões diminuem, sendo que para $n=5000$ e 10000 os valores são quase iguais. Para amostras de tamanho $\mathrm{n}=100000$ o método II foi bem mais preciso.

\section{CONSIDERAC̣ÕES FINAIS}

Os resultados das comparações entre os valores fornecidos pelos métodos paramétrico e não-paramétrico para a obtenção da constante $C_{R \alpha}$, para $\mathrm{p}=2$ e $\mathrm{p}=4$, mostram que a partir de amostras de tamanho $n=5000$ os dois métodos apresentam resultados muito semelhantes e próximos do valor real da constante, apresentados nos artigos de Hayter e Tsui (1994) e Bechhofer \& Dunnett (1988). Assim, em termos de implementação computacional, por requerer um tempo menor de execução, o algoritmo do método não-paramétrico (ver Figura 2) deve ser preferido para a determinação da constante $C_{R \alpha}$ quando se tem amostras de tamanhos $n$ maior ou igual a 5000. No entanto, em termos práticos nem sempre se dispõe de uma amostra tão grande do processo. É importante salientar que o método não-paramétrico não é

Tabela 2: Medidas descritivas das estimativas de correlação obtidas nas $\mathbf{N}=100$ amostras utilizadas no cálculo da constante $\mathbf{C}_{\mathbf{R} \alpha}$.

\begin{tabular}{|c|c|c|c|c|c|}
\hline $\begin{array}{c}\text { TAMANHO DA } \\
\text { AMOSTRA (N) }\end{array}$ & MÉDIA & DESVIO-PADRÃO & MÍNIMO & MEDIANA & MÁXIMO \\
\hline 50 & 0,5811 & 0,1033 & 0,2999 & 0,6004 & 0,7599 \\
\hline 100 & 0,59291 & 0,06490 & 0,37835 & 0,59731 & 0,73108 \\
\hline 500 & 0,59968 & 0,02995 & 0,53957 & 0,60118 & 0,66463 \\
\hline 1000 & 0,59847 & 0,01722 & 0,55166 & 0,60128 & 0,65144 \\
\hline 5000 & 0,60024 & 0,00809 & 0,57782 & 0,60098 & 0,61947 \\
\hline 10000 & 0,59875 & 0,00587 & 0,58549 & 0,59888 & 0,61160 \\
\hline 100000 & 0,60001 & 0,00203 & 0,59438 & 0,60026 & 0,60382 \\
\hline
\end{tabular}


Figura 11: Comparação dos métodos I e II para tamanhos de amostra $n=50,100,500$ e 1000. $p=4$ - $(\alpha=0,05)$.

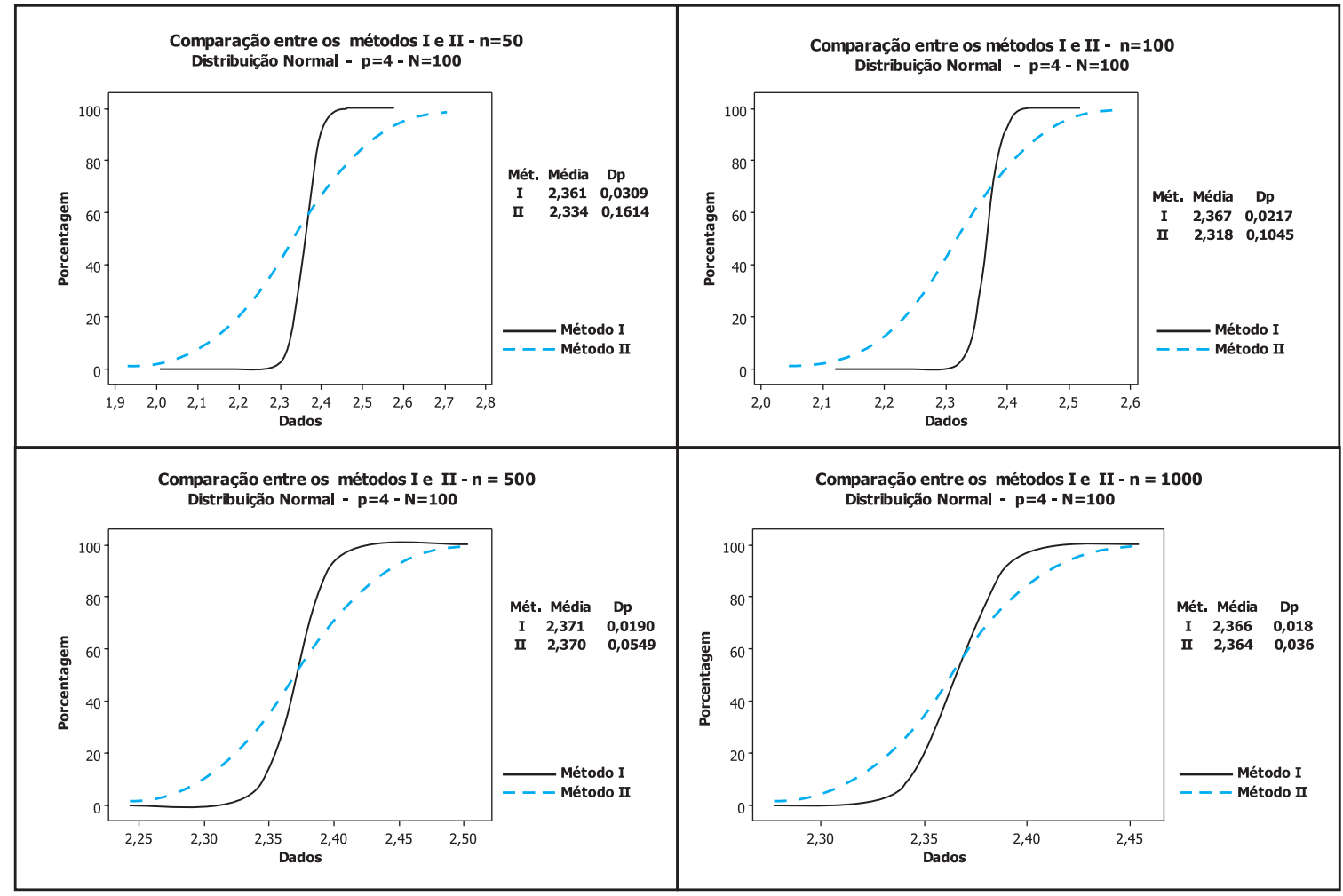

Figura 12: Comparação dos métodos I e II para tamanhos de amostra $n=5000,10000$ e 100000. $p=4-(\alpha=0,05)$.
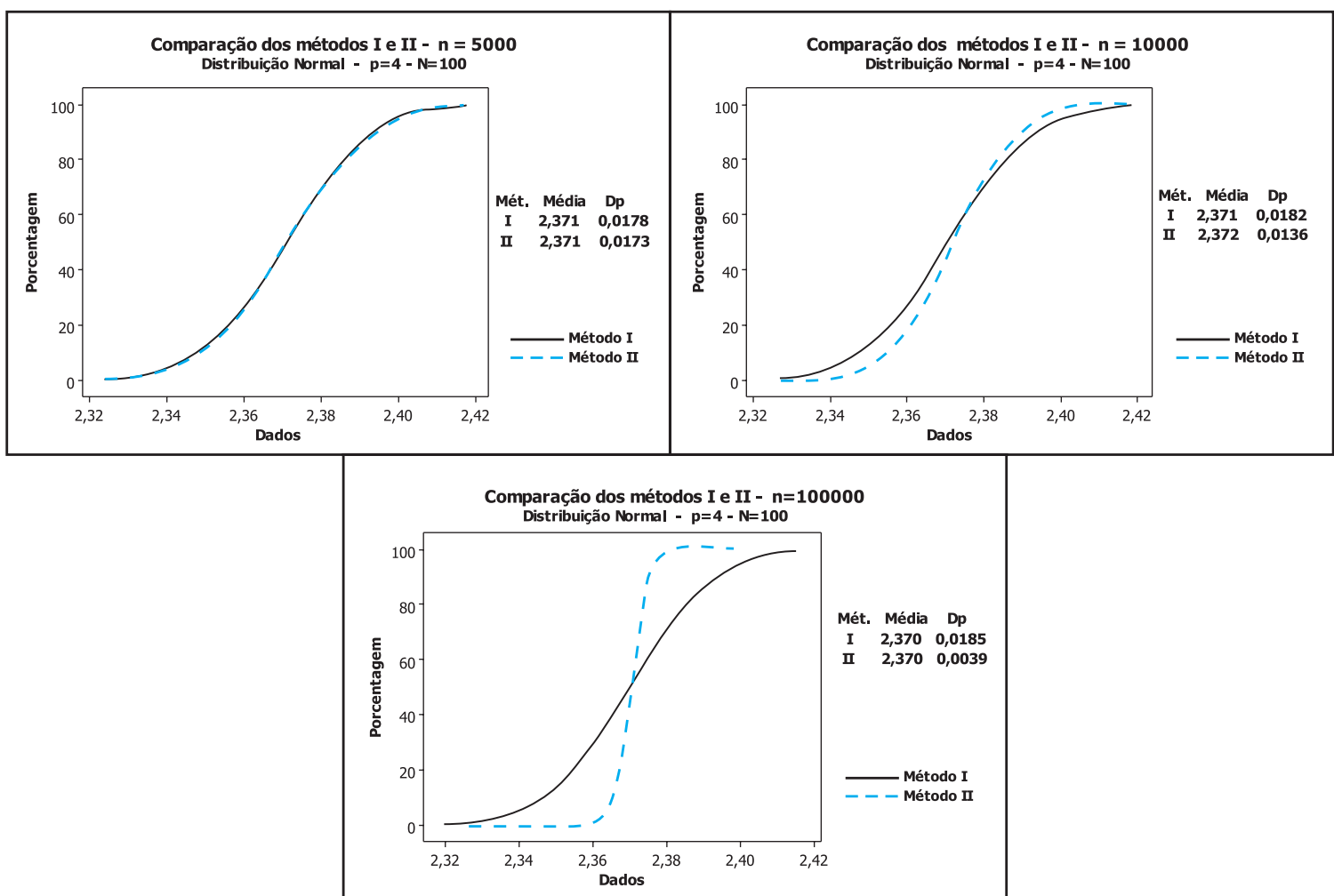
recomendável para amostras pequenas. Pode-se observar uma inversão no comportamento das funções de distribuições empíricas dos dois métodos conforme o aumento do tamanho da amostra, sendo que para amostras pequenas o método paramétrico é mais preciso. Além disso, este estu- do mostra que, no caso da distribuição normal, no valor $\mathrm{n}=500$ os dois métodos ainda diferem substancialmente, ao contrário do que foi sugerido por Hayter e Tsui (1994), que indicam o uso do método não-paramétrico para amostras a partir de $n=500$.

\section{Artigo recebido em 19/07/2004 \\ Aprovado para publicação em 10/06/2005}

\section{- Referências Bibliográficas}

ALT, F.B. Multivariate quality control. In Encyclopedia of Statistical Sciences 6, editado por S. Koltz e N. L. Johnson. Nova York: John Wiley \& Sons, 1985.

BECHHOFER, R.E., DUNNETT, C.W. Percentage points of multivariate student t-distributions. Selected Tables in Mathematical Statistics, 11 American Mathematical Society, Providence, RI, 1988.

CHEN, H. A multivariate process capability index over a rectangular solid tolerance zone. Statistica Sinica,vol. 4, p. 749-758, 1994.

COSTA, A.F.B., EPPRECHT, E.K., CARPINETTI, L. C. R. Controle Estatístico de Qualidade. São Paulo: Editora Atlas, 2004.
DOGANAKSOY, N.; FALTIN, F. W.; TUCKER, W.T. Identification of out of control quality characteristic in a multivariate manufacturing environment. Communications in Statistics-Theory and Methods, 20, p.1095-1111, 1991

DUNN, O. J. Estimation of the means of dependent variables. Annals of Mathematical Statistics, 29, p. 27752790, 1958.

JOHNSON, R.A. \& WICHERN, D.W. Applied multivariate statistical analysis. New Jersey: Prentice Hall, 2002.

JOHNSON, R.; BATTACHARYYA, G. Statistics: principles and methods. New York: John Wiley, 2001.

HAYTER, A. J.; TSUI, K-L. Identification and quantification in multivariate quality control problems. Journal of Quality Technology, v. 26, n. 3, p. 197208, 1994.
HOTELLING, H. Multivariate quality control. In Techniques of Statistical Analysis, editado por Eisenhart Hastay e Wallis. New York: John Wiley \& Sons, 1947.

MARDIA, K.V. Assessment of multinormality and the robusteness of Hotelling $T^{2}$ test, Applied Statistics, $24,163-171,1974$.

MASON, R.L., CHOU; Y-M, YOUNG, J. C. Applying Hotelling's $T^{2}$ statistic to batch processes. Journal of Quality Technology, vol. 33, n. 4, p. 466-479, 2001.

MASON, R.L., TRACY, N.D. YOUNG J.C. A practical approach for interpreting multivariate $T^{2}$ chart signals. Journal of Quality Technology v. 29 , p. 99-108, 1997 .
MASON, R.L.; YOUNG, J.C. Multivariate statistical process control with industrial applications. Pennsylvania: Siam and Alexandria: ASA, 2002.

MINGOTI, S.A.; GLÓRIA, F.A.A. Uma proposta de modificação do índice de capacidade multivariado de Chen. In Anais do XXIII ENEGEP, Ouro Preto, Minas Gerais, 2003 (em CD-ROM).

MONTGOMERY, D.C. Introdução ao controle estatístico de qualidade. Rio de Janeiro: Livros Técnicos e Científicos, 2004.

NOMIKOS, P.; MACGREGOR, J.F. Multivariate SPC charts for monitoring batch Processes. Technometrics,vol. 37, p. 41-59, 1995.

SIDAK, Z. Rectangular confidence regions for the means of multivariate normal distribution. Journal of American Statistical Association, 62, p. 626-633, 1967.

\section{- Agradecimentos}

Os autores deste artigo foram parcialmente financiados pelo CNPq. Os autores agradecem também aos dois revisores anônimos pelos valiosos comentários que auxiliaram na melhoria da qualidade desse artigo.

\section{Sobre o autor}

\section{Sueli Aparecida Mingoti}

Ph. D. em Estatística. Profa. Adjunta do Departamento de Estatística da UFMG.

E-mail: sueli@est.ufmg.br

Universidade Federal de Minas Gerais

Departamento de Estatística - ICEx.

Endereço: Av. Antonio Carlos, 6627 - Campus Pampulha

30 161-970 - Belo Horizonte - Minas Gerais.

Fones: (031) 3-4995948 ou 3-499-5924 (fax)

\section{Fernando Augusto Alves Glória}

Aluno do Curso de Mestrado em Estatística da UFMG.

E-mail: bodimufmg@yahoo.com.br 